\title{
IoT Based Bank Security System
}

\author{
J.S Vimali, Senduru Srinivasulu, Gowri. S
}

\begin{abstract}
Paper This paper proposed an effective watching and controlling system for bank locker rooms which is completely self-decision. The security system is planned to perceive the unlawful entrance in the bank locker room zones that ordinarily happens in cases of the thefts. The genuine stress with current physically managed security structure is that if the robbery occurs by then the banks are not had the ability to recognize the plunderers due to nonattendance of confirmation. In our investigation we use Microcontrollers with different sensors (PIR, Smoke or Fire) as observatory to recognize or perceive intruder or unpredictable activities inside the bank and ATM. The structure will focus on the security of the bank locker rooms in an effective course by recognizing and controlling unapproved development. The proposed security system will save the pictures at whatever point the development will be recognized that can be used in future for examination The system will pass on the image data reliably to the remote territory control rooms using web based checking through neighborhood (LAN) and can moreover send the notice message short message organization (SMS) to the manager using GSM technique.
\end{abstract}

Keywords: Fire Sensor, Camera, PIR Sensor, Wireless Transmitter.

\section{INTRODUCTION}

For a common individual the bank infers a spot which addresses a best component of security. Reliably we are drawn in with banking trade. To confirm our exorbitant pearls, basic reports or cash, we use to use bank locker rooms. It has transformed into a basic bit of our life. To get by in this forceful world and for a predictable improvement, the budgetary business needs to give an abnormal state of security. Because of the open interest every day new branches are opening. The more number of branches required more noteworthy security.

Current structures and organizations are ending up to a regularly expanding degree independent and the monetary region isn't unreasonably far from it. Video observation in moving domains has transformed into a present topic of energy for PC vision development. You can see all the branches are under the perception of CCTV cameras, alert systems, emergency gets, etc. The CCTV cameras are used to screen the unapproved activity. It ought to be watched reliably by an individual which is troublesome work; especially in nighttimes. The alert emergency get moreover needs to be pressed physically. This conventional structure requires some portion of work. A structure can be made which will customized recognize unapproved development

Revised Version Manuscript Received on 10 September, 2019.

J.S Vimali, School of Computing, Sathyabama Institute of Science and Technology, Chennai, Tamilnadu, India

SenduruSrinivasulu, School of Computing, Sathyabama Institute of Science and Technology, Chennai, Tamilnadu, India.

Gowri.S, School of Computing, Sathyabama Institute of Science and Technology, Chennai, Tamilnadu, India. and instruct to the security experts concerning the banks by different ways with no need of a person.

The Microcontroller Based Bank Security System fulfills all these necessities. A model of this security structure has been arranged in the composition to extend the component of security in bank locker rooms enough. The development area will be done through camera itself and the hardware related with it will give unmistakable ways to deal with light up the security experts for instance using alert system a notice message and the image which has recognized the development will be normally exchanged on page which can be downloaded from wherever. For illuminating a GSM module will be utilized. The fundamental point of this examination is to structure a framework for alarming burglary and to auto capture the criminal in bank or ATM itself from brought together checking unit. The motivation behind the framework is to plan a savvy and concentrated checking and control framework utilizing IOT advances. The basic objectives of bank security system are following the bank locker room locales, acknowledgment of development and making the principal control move. The further portions will depict that how these objectives have been practiced.

\section{RELATED WORK}

The current surveillance systems and services are automated and the banking sectors are also moving towards get more automated in every aspect of security layers in the bank. In computer vision technology, video surveillance is having high demand, and a current topic of interest. These days all the security layers are under the surveillance of CCTV cameras, alarms systems, emergency buttons etc. The CCTV cameras are used to monitor and capture the unauthorized movements and activities. The surveillance needs a human intervention continuously irrespective of day and night, A very strict and vigilant human intervention for $24 * 7$ is very difficult task. Most of the security layers and the alarms need human intervention (need to be pressed manually).The traditional systems need more human intervention more man power. To overcome the security flaws and minimize the man power, we need a new system that continuously monitors and detects the unauthorized movements and alters the security officials of the bank by various ways without any human intervention. The best option is to make use of the microcontroller Based Bank security System that takes care of all the flaws which usually happen in conventional systems. A new paradigm for security system has be designed to effectively enhance and increase the level of security especially in more high secured areas like bank locker rooms. Any unauthorized motion 
detected in the high secured zone will be monitored by CCTV cameras and immediately intimated to higher officials by the three different ways. Automatic alarm system, automatic warning message to mobile devices and the unauthorized motion detected is captured as image and will be automatically uploaded in the official cloud enabled web page, which can be downloaded from anywhere any time and any device .For automatic messaging a GSM module is used. The main objective of the bank security system is to give high proof security to high secured zones like bank locker rooms, detection of au authorized movements and initiating a quick action.

A couple tasks have been done on Smart security structures till date. Examiners these days have thought of modifications to the unrefined structures proposed previously. The most key idea being the social occasion of an alert message on intruder disclosure. Here a PIR sensor has been used for recognizing development and a GSM module to send message alerts to the customer. At any rate there is no information about the interloper and fails to assemble verification. Another examination outfits its customers with a of emergency conditions. The system including a furor get is set at a region adequately gotten to by the customer, which when crushed sends a pre-saved message to the police. The limitation of this system is that the customer needs to physically press the catch in the midst of emergency and proposes a structure which is fit for watching sensors and controlling actuators like robots, Unmanned Aerial Vehicles (UAVs) for home security systems. This system shows higher execution and is totally robotized, at any rate ends up being in all regards costly to continuous utilization in private nuclear families or on a smaller scale. Various distinctive examinations use Bluetooth, Light Dependent Resistors and Zigbee headways for home robotization and security, at any rate these advances are not as secure as $\mathrm{Wi}-\mathrm{Fi}$ and have Wireless Personal Area Network (WPAN) controls, as incorporation region is limited for Bluetooth. The most outrageous data conversion scale of Zigbee is lower than minimal rate of $\mathrm{WiFi}$.

\section{EXISTING SYSTEM}

The framework contains a sensor to distinguish movement. The entire framework is constrained by an 8051 smaller scale controller Atmeg32. It gathers data from the sensors settles on a choice and sends SMS to a comparing number by utilizing a GSM modem. In the event that it finds any interference in its sensors like in the event that the IR is intruded on, at that point PIC will send a SMS to the mortgage holder. The disadvantages are: GSM based technology used only for alerting purpose. Only one sensor is used for security identification purpose alone. It supports only low level security system. Take too much time to execute process in gsm technology. Low speed data communication micro controller is used.

\section{PROPOSED SYSTEM}

The microcontroller considers the sensor regards. In case the intruder was perceived the information will be securely fundamental and brisk way to deal with get help in the midst structure does not recognized. One other examination

arranged and checked through central dealing with core interest. By using this method minute watching and control was possible. No convincing motivation to screen reliably and less human was related with checking territory. The advantages are: IOTbased Technology is used, High speed data communication process, Multiple sensors used for advantage monitoring, Automatic image arresting and immediate alerting system and Live monitoring from anywhere and anytime using IOT technology. PIC microcontrollers are a group of specific microcontroller chips delivered by Microchip Technology in Chandler, Arizona. The abbreviation PIC means "fringe interface controller," in spite of the fact that that term is once in a while utilized these days. A microcontroller is a minimized microcomputer intended to administer the activity of implanted frameworks in engine vehicles, robots, office machines, therapeutic gadgets, portable radios, candy machines, home apparatuses, and different gadgets. An average microcontroller incorporates a processor, memory, and peripherals.

\section{IMPLEMENTATION\& RESULTS}

This zone will layout about the exceptional techniques picked to realize the safety system and the work procedure of the structure. The sector will moreover appear unmistakable program codes used for the utilization of the system.

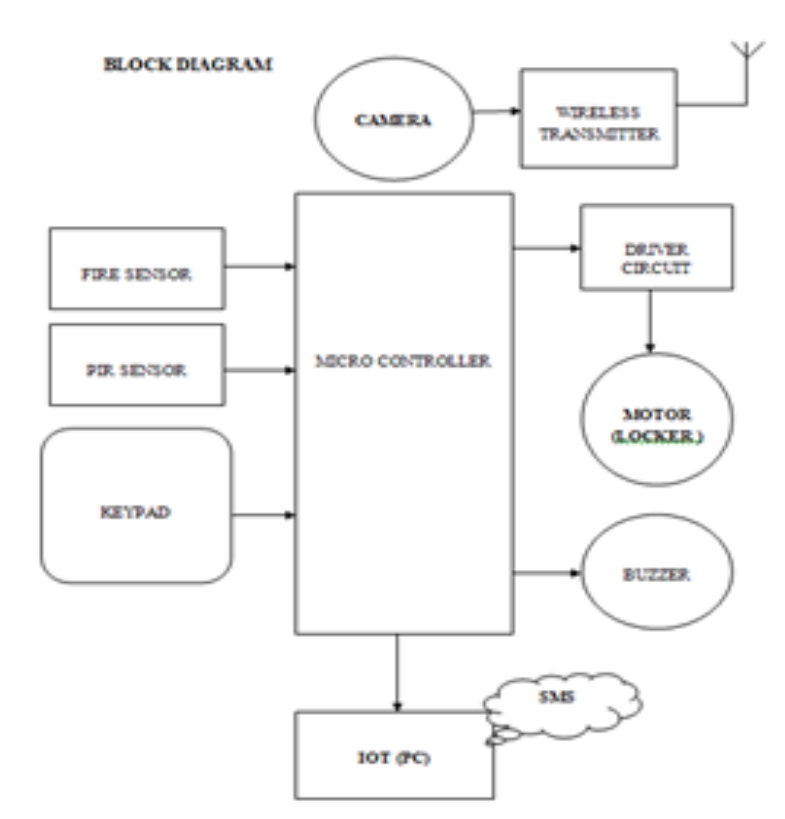

Fig. 1.1: Block Diagram

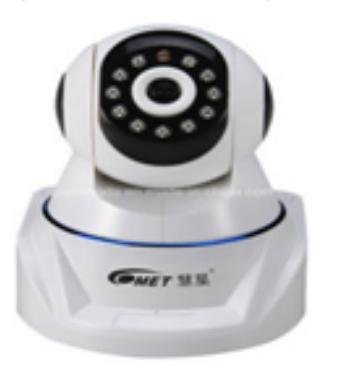

Fig.1.2: Wireless Camera

Published By: Blue Eyes Intelligence Engineering 


\section{A. CENTRAL PROCESSOR UNIT}

A microcontroller (or MCU for microcontroller unit) is a little PC on a lone composed circuit. In present day wording, it resembles, anyway less mind boggling than, a system on a chip or SoC; a SoC may join a microcontroller as one of its fragments. A microcontroller contains somewhere around one CPUs (processor-focuses) close by memory and programmable data/yield peripherals. Program memory as ferroelectric RAM, NOR flash or OTP ROM is moreover routinely included on chip, similarly as a little proportion of RAM. Microcontrollers are planned for embedded applications, as opposed to the microchips used in PCs or other generally helpful applications containing distinctive discrete chips.

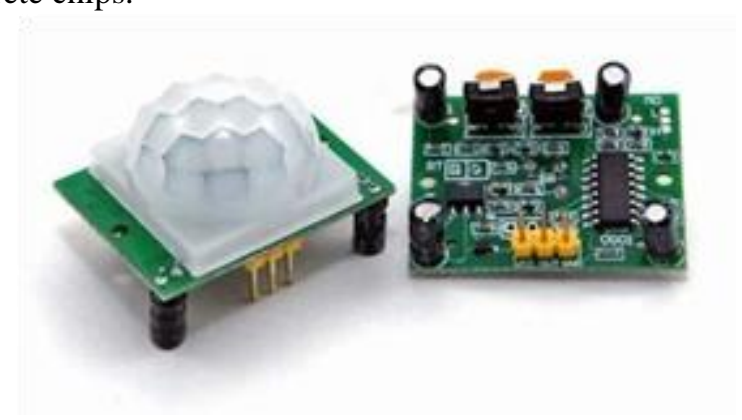

Fig.1.3: PIR Sensor

PIR sensors are more puzzled than an extensive parcel of interchange sensors cleared up in these instructional activities (like photocells, FSRs and tilt switches) in light of the fact that there are different variables that impact the sensors data and yield. The PIR sensor itself has two spaces in it, each opening is made of a phenomenal material that is tricky to IR. The point of convergence used here isn't by and large doing much therefore we see that the two spaces can 'see' out past some partition (basically the affectability of the sensor). Right when the sensor is idle, the two openings recognize a comparative proportion of IR, the encompassing whole radiated from the room or dividers or outside. Right when a warm body like a human or animal travels by, it first gets one segment of the PIR sensor, which causes a positive differential change between the two sections. Right when the warm body leaves the recognizing zone, the modify happens, whereby the sensor makes a negative differential change. These change beats are what is recognized.

\section{B. SYSTEM SENSING UNIT}

It is a system that changes over a straightforward banner into a mechanized banner and it gives an isolated estimation, for instance, an electronic device that changes over a data basic voltage to a propelled number comparing to the span of the voltage. A sensor is a contraption that perceives and responds to a type of commitment from the physical condition. The specific information could be light, heat, development, sogginess, weight, or any of an amazing number of other normal wonders.

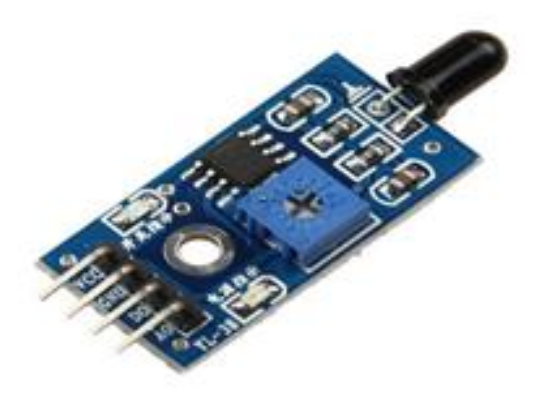

Fig.1.4: Fire Sensor

This flame sensor circuit misuses the temperature detecting property of a conventional flag diode IN 34 to identify heat from flame. Right now it detects heat; an uproarious caution recreating that of Fire detachment will be delivered. The circuit is excessively delicate and can distinguish an ascent in temperature of 10 degree or more in its region. Common flag diodes like IN 34 and OA 71 shows this property and the interior obstruction of these gadgets will diminish when temperature rises.

\section{C.SERIAL COMMUNICATION}

Comprehensive Asynchronous Receiver/Transmitter is a touch of PC gear that translates data among parallel and successive structures In media transmission and data transmission, successive correspondence is the route toward sending data one piece at some random minute, sequentially, over a correspondence channel or PC transport. This is rather than parallel correspondence, where a couple of bits are sent all things considered, on an association with a couple of parallel channels.

\section{CONCLUSION}

This paper demonstrates an insignificant exertion, shrewd and confirmed structure for the bank locker room area. The structure will focus on the prosperity of the bank locker rooms in a practical way by distinguishing and controlling unapproved development. The planned safety system will distinguish the development through camera and gives security by taking three unmistakable control exercises. In this system visual perception estimation has been completed for development recognizable proof on the host PC and the consequent picture data has been conferred to client side PCs for electronic checking through neighborhood mastermind (LAN). The unapproved picture recognizable proof banner is also online granted to the microcontroller that sets the forewarning ready close-by furthermore send the notice message short message organization (SMS) to the executive using GSM framework for taking critical control exercises. The proposed sharp structure has central focuses over the present security system like insignificant exertion, stable association, significant incorporation domain, and low correspondence cost. 


\section{FUTURE ENHANCEMENT}

The future upgrade to this work should be possible by including some more viewpoints, for example, face acknowledgment. Along these lines it improved the dependability of bank locker and unapproved access will be limited. The upgrade could be additionally connected to recognize the unlawful passage.

\section{REFERENCES}

1. Robert T. Collins, Alan J. Lipton, Hironobu Fujiyoshi, and Takeo Kanade, "Algorithms For Cooperative Multisensor Surveillance," In Proc of the IEEE, vol. 89, no. 10, Oct. 2001, pp. 1456-1477.

2. PiyushMayank and SudiptaMukhopadhyay, "Temporal Correlation and Probabilistic Prediction Based Face Detection Framework in Real Time Environment," IEEE Proceedings of 4th International Conference on Intelligent Human Computer Interaction, Kharagpur, India, December 27-29, 2012.

3. Weiming $\mathrm{Hu}$, Tieniu Tan, Liang Wang, and Steve Maybank, "A Survey on Visual Surveillance of Object Motion and Behaviors," in Proc. of IEEE Transactions on Systems, MAN, and Cybernetics vol. 34, no. 3, Aug. 2004, pp. 334-351.

4. Sadeque Reza Khan, "Development of Low Cost Private Office Access Control System(OACS)," International Journal of Embedded Systems and Applications, vol.2, no.2, June 2012.

5. A. Alheraish, "Design and Implementation of Home Automation System," IEEE Transactions on Consumer Electronics, vol. 50, no. 4, Nov 2004, pp. 1087-1092.

6. Ma Yuchun, Huang Yinghong, Zhang Kun and Li Zhuang, "General Application Research on GSM Module," in Proc, of International Conference on Internet Computing and Information Services, , pp.525- 528,2011.

7. V. Vinoth Krishnan, M. Saianand, J.S.Vimali, "RESPONSE MECHANISM FOR CRISIS TIMES FROM SOCIAL NETWORKS", International Journal Of Pharmacy \& Technology (IJPT), June-2016, Vol. 8 | Issue No.2, Pages:11958-11966.

8. N.Jerry Joshua, M.Gowtham, J.S.Vimali, "A Proactive Advance For Dynamic, Optimal Scaling in a Geo-Distributed Cloud", International Journal Of Pharmacy \& Technology (IJPT), June-2016, Vol. 8, Issue No.2, Pages: 3797-3807. 